\title{
Digital Engineering Sensor Architectures for Future Microreactor Builds
}

Paul Plachinda

Chris Ritter

Piyush Sabharwall

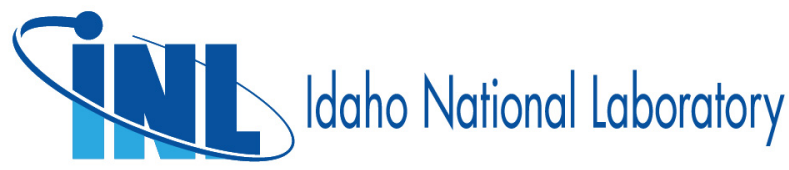




\section{DISCLAIMER}

This information was prepared as an account of work sponsored by an agency of the U.S. Government. Neither the U.S. Government nor any agency thereof, nor any of their employees, makes any warranty, expressed or implied, or assumes any legal liability or responsibility for the accuracy, completeness, or usefulness, of any information, apparatus, product, or process disclosed, or represents that its use would not infringe privately owned rights. References herein to any specific commercial product,

process, or service by trade name, trademark, manufacturer, or otherwise, does not necessarily constitute or imply its endorsement, recommendation, or favoring by the U.S. Government or any agency thereof. The views and opinions of authors expressed herein do not necessarily state or reflect those of the U.S. Government or any agency thereof. 


\section{ACKNOWLEDGEMENT}

This material is based upon work supported by the U.S. Department of

Energy, Office of Nuclear Energy. The authors would like to acknowledge Prof. Gupta and Prof. Han of North Carolina State University for their support during this study. 
Page intentionally left blank 


\begin{abstract}
The development of new nuclear reactors continues to be high risk during the construction phase, with many programs exceeding their budget by $2-3 \times$. This work entails an investigation of sensors that can be utilized during the construction of microreactors and their incorporation into a digital twin supporting the construction process. These sensors can be used to monitor construction progress, inform of variances from the expected design, and verify that the reactor is being built to the planned building information management model. This will ensure reactor equipment and components accurately interact through welding or additive manufacturing technologies. This study illustrates the importance of the digital engineering architecture for microreactor deployment.
\end{abstract}


Page intentionally left blank 


\section{CONTENTS}

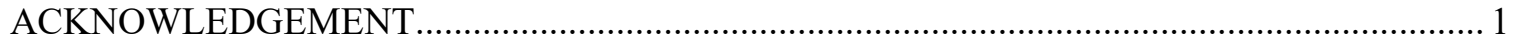

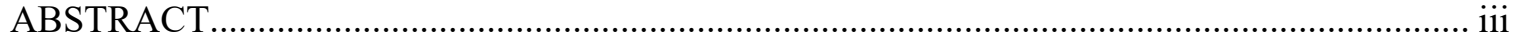

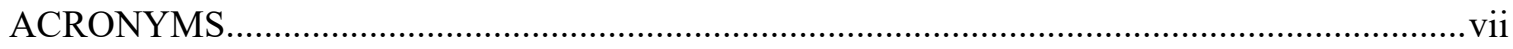

1. THE IMPERATIVE FOR DIGITAL TWIN IN MICROREACTOR CONSTRUCTION.... 1

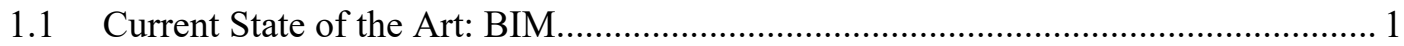

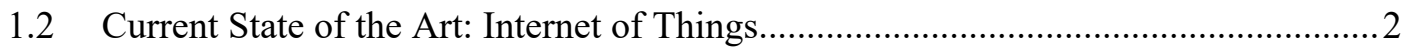

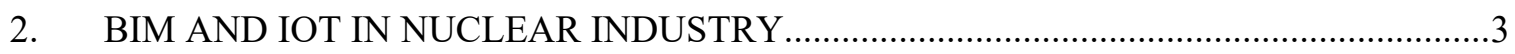

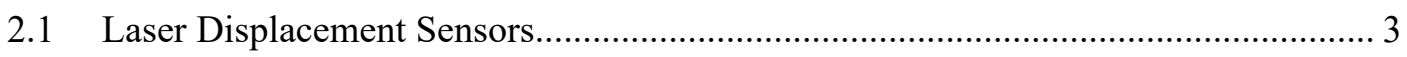

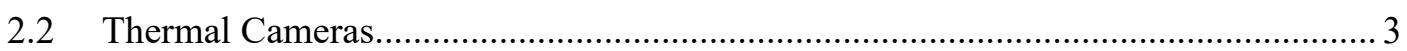

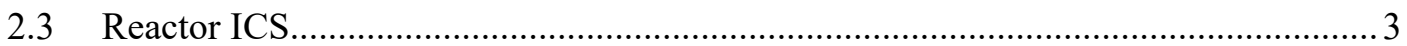

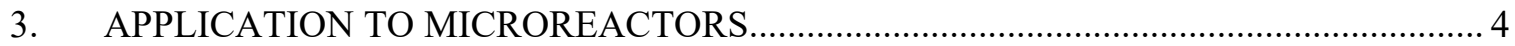

3.1 Sensor Instrumentation of a Micro Reactor................................................................... 5

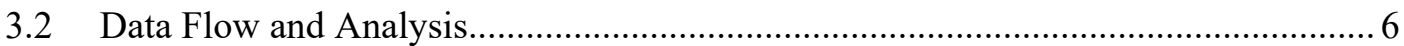

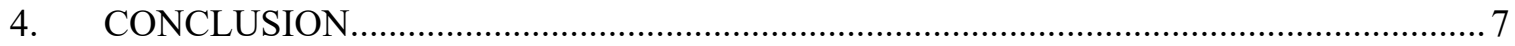

\section{FIGURES}

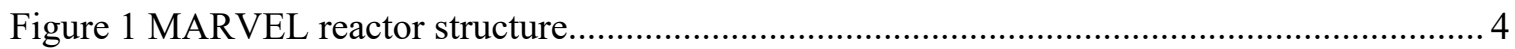

Figure 2. Annular-axial (CT) gantry. And the Reactor instrumentation concept........................... 5

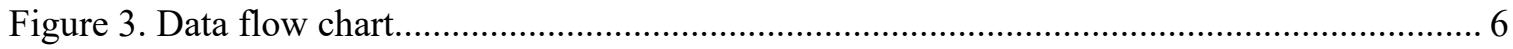


Page intentionally left blank 


\section{ACRONYMS}

BIM building information management

CAD computer-aided design

DT digital twin

FoV field of view

GPS Global Positioning System

ICS instrumentation and control system

INL Idaho National Laboratory

IoT internet of things

LDS laser displacement sensor

LIDAR light detection and ranging

MARVEL Microreactor Applications Research, Validation and Evaluation

SCS secondary containment structure

SLAM simultaneous localization and mapping 
Page intentionally left blank 


\section{Digital Engineering Sensor Architectures for Future Microreactor Builds}

\section{THE IMPERATIVE FOR DIGITAL TWIN IN MICROREACTOR CONSTRUCTION}

The development of new nuclear reactors continues to be high risk during the construction phase. More than $53 \%$ of typical construction projects are behind schedule, and more than $66 \%$ suffer from cost overruns (Bevan and Steve 2016). The main technical and project management risks during the construction phase stem partly from an inability to accurately capture the construction progress, including delays in putting together critical structural elements of the reactor facility, deviations from the approved plan and engineering drawings (i.e., failed verification), and errors accumulated during the design process (i.e., failed validation). Digital engineering is meant to address these issues by establishing an authoritative source of truth and to support the continuous monitoring of the construction process, operation of the reactor, and the decommissioning and decontamination of that reactor. The objective of this study is to emphasize the importance of digital engineering for microreactor construction.

The construction process can be captured in real time if various sensors monitoring the construction progress and current (even if intermediate) state of the containment structure are incorporated into the design and are installed while the containment is being built. The benefit of this approach is that a digital twin of a building emerges ex processus the construction, allowing for a dynamic capture of it.

This scope would look into sensors that can be utilized during the construction of microreactors and their incorporation into a digital twin of the construction process. These sensors can be used to monitor the construction progress, discover variances from the expected design, and verify that the reactor is built to the planned building information management (BIM) model. This will ensure reactor equipment and components accurately merge through welding and additive manufacturing technologies.

\subsection{Current State of the Art: BIM}

With an ever-increasing amount of visual data available on construction sites due to advances in computer vision and 3D imaging technologies, there have been dramatic advances in model-based construction progress detection leveraging as-built modeling techniques. Some of these techniques use image-based point clouds. Other techniques use laser-scanned point clouds.

Image-based point clouds are usually obtained by flying a camera-equipped drone along the same predefined path and taking a stream of images of the construction site. Nowadays, it is common to capture hundreds of images of a jobsite on a daily basis. (Ham et al. 2016; Han and Golparvar-Fard 2017). Laserbased point clouds are collected using light detection and ranging (LIDAR). Laser-scanned methods are based on geometry recognition and generally provide more accurate and denser point clouds of the structures of interest than the image-based methods. The downside, however, is that the LIDAR is usually significantly heavier than cameras, $\sim 1.5 \mathrm{~kg}$ versus $\sim 50 \mathrm{~g}$. This increased weight puts an extra burden on a drone's battery, compromising its fly time and thus its range. Therefore, most laser scan jobs are performed using surface-based devices, often moved across the jobsite manually or using terrestrial vehicles. This approach requires a manual intervention to define a path and is hindered by unexpected obstacles appearing along the path. 
The data collected using either of the methods (as-built model) is then correlated automatically with the BIM information (as-planned model). Both image-based and laser-based methods deliver accurate information about the geometry of the structure; however, the image-based method can also reveal textures of different BIM elements. The image-based 3D reconstruction process consists of structure from motion for sparse reconstruction (Wu 2017) and multiview stereo for dense reconstruction (Goesele et al. 2007). Images are the inputs to this pipeline of structure from motion to multiview stereo and camera poses (intrinsic and extrinsic camera parameters) and point clouds are the outputs. On the other hand, the 3D laser scanners typically used in construction sites (e.g., time-of-flight terrestrial laser scanners) are used to generate 3D point clouds with commercially available software. Corresponding features (e.g., corners) between point clouds and BIMs are manually picked and similarity transformations are applied to register the as-built and as-planned models and create an integrated planning model. The data collected at this step is then filtered based on the geometry available from the BIM (e.g., images taken by drones tend to capture background objects that do not belong to the structure of interest). The next step, appearance-based reasoning, classifies material classes of the detected BIM elements based on color and texture. More details on these approaches can be found in (Han, Degol, and Golparvar-Fard 2018).

In many cases, laser-based data collection is still conducted manually. To address this limitation, the SLAM (Simultaneous Localization and Mapping) technique has been developed by (Engel et al. 2014 and others). SLAM is an algorithmic attempt to address the problem of building a map of an unknown environment while navigating the environment using the map. Mapping the spatial information of the environment is done on-the-fly with no prior knowledge of the robot's location. The built map is subsequently used by the robot for navigation. Most external SLAM applications use Global Positioning System (GPS) shortcutting to acquisition location information. Of course, poor satellite signal coverage for indoor environments limits the GPS accuracy. 3D LIDAR scanners have limited efficiency in large scale environments; there will be areas lacking in features discernible by a laser, like open hallways or glass-walled corridors. Processing time has also historically limited the speed at which 3D LIDAR point clouds can be registered. Some of these limitations are addressed in (Asadi et al. 2019). This paper describes a method that performs an automatic registration of image sequences to BIM in real time, leveraging BIM's geometry and computer vision techniques, such as camera localization and mapping and perspective detection.

\subsection{Current State of the Art: Internet of Things}

Another aspect of digitalization of construction is use of the internet of things (IoT). Recent tremendous progress in semiconductors and communication enables real-time data collection and aggregation from thousands of sources. IoT enabling technologies include advanced sensing, pattern identification and recognition, cloud computing, communication technologies, and networks.

The potential of connecting BIM and IoT-based data sources is a relatively new development. As a generalization, BIM and IoT data offer complementary views of the project that together supplement the limitations of each. BIM data contains periodically refreshed geometric or materials data from the entire construction site, whereas sensors are usually placed relatively sparse (as compared to the point cloud used for BIM), but they deliver information inaccessible for BIM scanners. Thus, IoT data enhances BIM geometric and assets information set by providing a real time and recordable status from the actual operations in construction and operations. The information sampled from sensors is highly variable but includes positioning information, physical measurements, weather, etc. Positional information is provided via either an automated position determination (e.g., GPS) or manually via assigning a position to a sensor within the BIM.

Sensor data is generally organized as time series data streams of individual sensor point samples over time, frequently with some higher-level organization into equipment, asset, etc. Both BIM and IoT data may be accessed through similar mechanisms, including the manual interfaces of proprietary systems, Application Programming Interfaces associated with these applications, potential database connections to the systems, and export via open standards. This database allows to maintain one of the core features of BIM - a single source of truth that combines data from both BIM and IoT worlds. 


\section{BIM AND IOT IN NUCLEAR INDUSTRY}

Nuclear industry requirements are far more strict than general construction. The nuclear instrumentation and control systems are designed to have multiple redundancies and provide accurate measurements throughout the entire reactor lifetime while operating in a harsh environment (temperature, pressure, radiation, aggressive media, etc.). Reactor vessels, confinement, and other structural support elements of the reactor assembly are critical to ensure the integrity and containment of nuclear fuel and fission products. Constant in-situ monitoring of the "health" of the reactor core is performed by the instrumentation and control system (ICS), usually including thermocouples and flow meters, which are air monitor sensors to detect radionuclides. However, historically, little attention has been given to the initial health and cursus morbi of the containment structure.

Here, we will discuss the mesoscale concept that combines BIM (macroscale) and IoT (microscale) approaches to assess the as-built and as-evolved state of the containment structure. This will ensure that reactor equipment and components accurately merge together through welding and via additive manufacturing technologies and that this merger remains constant throughout the expected lifetime of the reactor.

\subsection{Laser Displacement Sensors}

Laser displacement sensors (LDS) can be employed to assess the reactor vessel geometry at both the manufacturing location as well as at the installation location. Laser displacement measurement systems provide non-contact, high-speed, and high-precision measurement. Unlike the cameras, LDS do not compromise accuracy in complex surfaces and geometries, where the picture may be distorted by shadowing. LDS have many applications in building technology; a method to measure building vibration has been proposed in (Bougard and Ellis 2000). The requirements and specifications of the LDS vary depending on the application. For example, a commercial 2D/3D laser scanner equipped with a blue laser can have $Z$ (axial) resolution as low as $3 \mu \mathrm{m}$ at a (lateral) field of view (FoV) of 3-4 mm or up to $500 \mu \mathrm{m}$ at a FoV 500-800 mm. (AccuProfile 820 series). A 3D scan is possible when a 2D sensor moves along the $\mathrm{Y}$ axis across the entire surface.

\subsection{Thermal Cameras}

Structural deviations from the ideal model can be broken down into several categories: poor welding or casting quality, poor material quality, and incorrect operation regimes. Poor welding or casting quality can be detected immediately whether at the place of manufacturing or at the place of installation. This is done via a scan using a hand-held x-ray apparatus to detect cavities in weldment seams, and an LDS scan to detect part deformation. Scan results are then compared with the computer-aided design (CAD) model of the weldment. Poor material quality can manifest itself in a slow deformation under constant stress (creep). Creep is accelerated under elevated temperatures caused by tougher than normal or incorrect operating regimes. Therefore, it is important to monitor the temperature distribution on the outer surface of the secondary containment structure (SCS). Commercially available thermal cameras provide real-time images with $640 \times 480$ resolution (307,200 pixels), $25 \times 19$ degree angular FoV, and thermal sensitivity less than 100mK (FLIR T640). Thermal cameras are equipped with lenses of different focal distances; therefore, the lateral FoV depends on the focal distance, feasible distance between the SCS, and the instrument. It is likely that a thermal camera must be put on a moving gantry, similarly to the LDS. It is important to notice that both the LDS and thermal camera are reflection-based instruments - that is, the detector can be placed in proximity to the source, unlike an x-ray, which is transmission based, and hence the detector should be placed on the opposite side of the source.

\subsection{Reactor Instrumentation and Control System (ICS)}

Whereas the thermal cameras can provide information about the temperature filed on the outside of the reactor vessel, the reactor's core ICS can deliver data from the inside of the SCS. This data is usually sparse and limited to the number of thermocouples installed within the SCS volume; however, it can 
detect early variations before their detrimental effects propagate to the SCS weldment and compromise the structural integrity of the confinement.

\section{APPLICATION TO MICROREACTORS}

In this report, we will focus on applications geared towards assessing the state of a microreactor. Microreactors are compact reactors that will be small enough to transport by truck. Microreactors could help solve energy challenges in a number of areas and normally produce less than 20-30 $\mathrm{MW}_{\text {th }}$. These reactors have a lower overnight capital cost due to their factory production, decreased construction complexity, one-time certification, and economies of scale. In this report, we will use the Microreactor Applications Research, Validation, and Evaluation (MARVEL) project under the DOE-NE Microreactor Program and the National Reactor Innovation Center as an example of how BIM and IoT can be applied to a new reactor design. MARVEL is a $100 \mathrm{kWth}(\sim 20 \mathrm{kWe})$ Stirling Engine microreactor, which makes it similar albeit smaller in scale to other microreactors, such as Oklo's Aurora (1.5 MWe) or the NuGen Engine (1-3 MWe). However, the approach outlined here is fully scalable to even bigger projects, such as TerraPower's Natrium (345 MWe) or X-Energy's Xe-100 (80-320 MWe).

MARVEL will have liquid sodium potassium eutectic as the primary coolant, with temperatures around $500^{\circ} \mathrm{C}$. The reactor control systems will consist of four independent vertical control drums and a central shutdown rod. The microreactor is expected to become operational in 2024. (Arafat 2020) describes the MARVEL project goals and the microreactor design. The external design of MARVEL is presented in Figure 1.

The outer shell of a microreactor vessel consists of a welded SCS resting on the reactor support frame, immersed in the reactor pit. The axial size of the SCS is approximately 3 meters, radial size- -1.5 m.

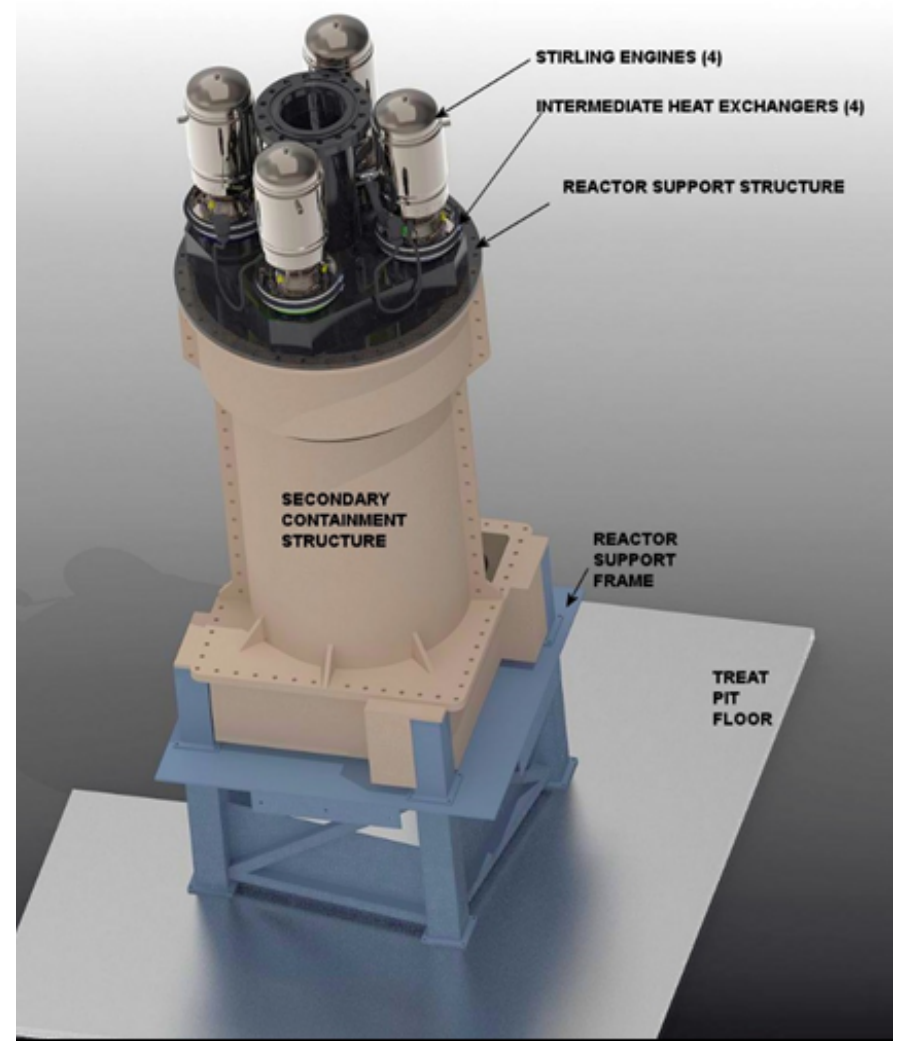

Figure 1. MARVEL reactor structure. 


\subsection{Sensor Instrumentation of a Microreactor}

The goal of this application is to construct a mesoscale real-time digital twin (DT) of MARVEL using the data coming from an array of sensors. Since, as described above, the FoV of the sensors is limited, it is important to utilize spatial scanners to cover the entire surface of the SCS. The objective is to capture the lateral deviation of the SCS vessel from the ideal CAD model and to monitor how these dimensions change throughout the lifetime of the reactor. Since the deviations could be in the microns order of magnitude, two types of gantry's geometry can satisfy this request: orthogonal gantries attached to the walls of the reactor pit or cylindrical gantries (annular-axial or tomographic) surrounding the reactor vessel. The orthogonal gantry has the advantage of being simple; however, a major drawback is that the working distance of cameras or an LDS changes with the axis' lateral position, and thus, it compromises resolution depending on the viewing angle. The annular-axial gantry (see Figure 2) would be free from these defects. There are many numerically effective reflective tomography approaches to reconstruct a 3D model of a shell from the $(\Theta, \mathrm{z})$ coordinates and signal intensity, see (Kadu, Mansour, and Boufounos 2020).

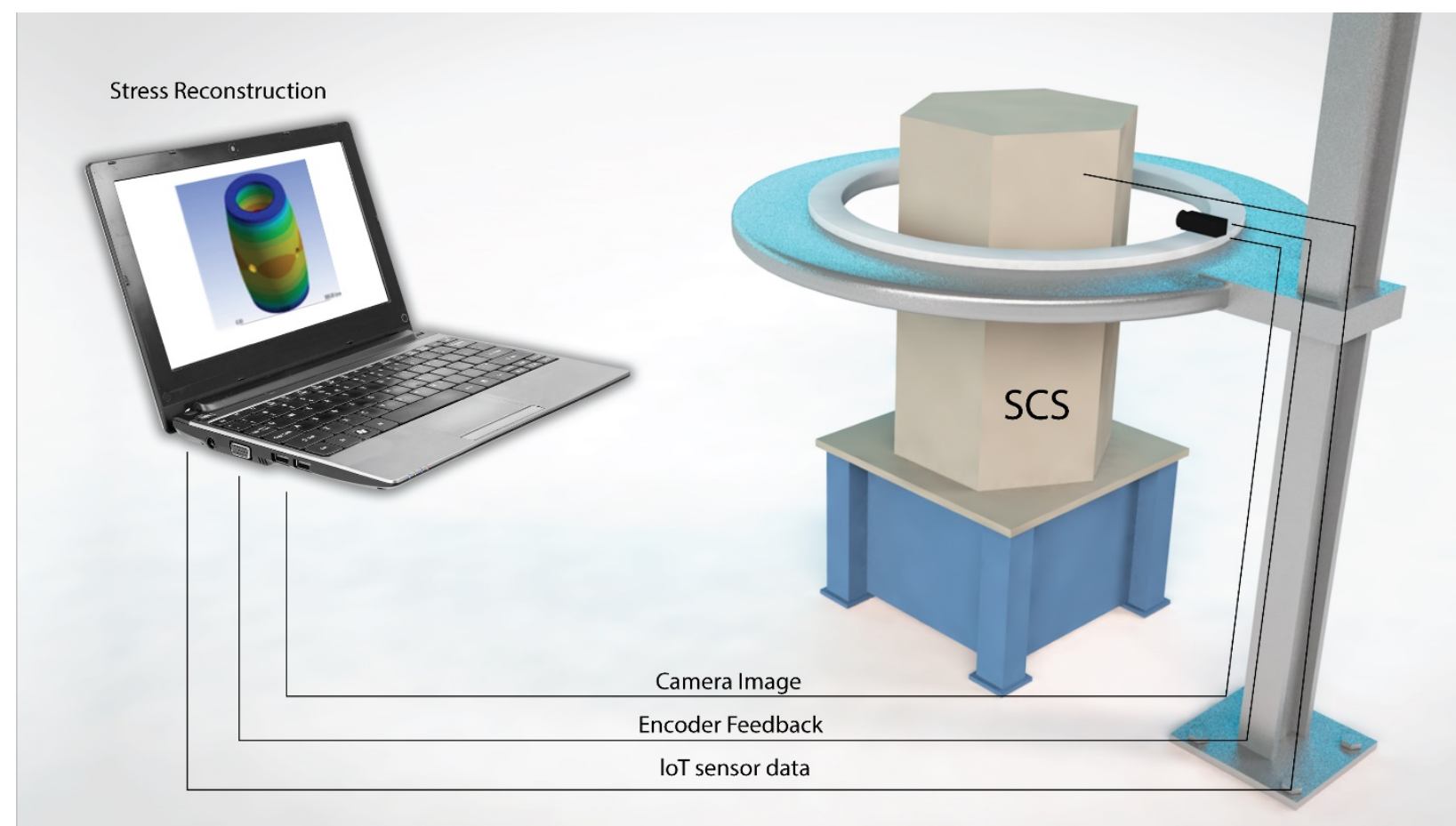

Figure 2. Annular-axial ("tomographic") gantry and the reactor instrumentation concept.

The result of such a reconstruction will be a DT of the SCS depicting temperature and displacement (strain) gradients over the SCS. Existing CAD models augmented with the DT data allow for backcalculating the total von Mises stress and comparing it with the yield stress of the SCS material to predict the rupture of the SCS.

Stainless Steel - Grade 316, commonly used in reactor confinement construction, has a thermal expansion coefficient of $15 \times 10^{-6} / \mathrm{K}$. The radial thermal expansion of a cylindrical structure is given by: $\mathrm{d}_{1}=\mathrm{d}_{0}(\Delta \mathrm{T} \alpha+1)$. Therefore, a camera with a thermal sensitivity of $100 \mathrm{mK}$ will be capable of detecting $\mathrm{a} \sim 2.5 \mu \mathrm{m}$ variance in the SCS diameter.

This data acquisition process should not be limited to the SCS weldment. If the spatial geometry permits, the power generating system and heat rejection system's external shells should also be monitored to prevent failures due to overheating. 


\subsection{Data Flow and Analysis}

With all of the advantages that the LDS and thermal camera monitoring systems deliver, they have a major drawback - non-real-time data collection, that is the scan process must be initiated automatically or manually. This is a common problem for all BIM data acquisition systems. Every time the CAD data is refined by the LDS scans to account for growing imperfections arising from manufacturing or operation defects, it forms a point cloud, a representation method common in BIM.

Here we are proposing to augment high-fidelity, high-resolution, high-coverage BIM-type data with sparse, but real-time, IoT data. The reactor core instrumentation includes a variety of sensors, such as the primary coolant flow meters, primary coolant level probes, primary coolant leak detectors, guard vessel pressure sensor, downcomer thermocouples, reflector thermocouples, intermediate heat exchanger thermocouples, lower plenum thermocouples, upper plenum thermocouples, neutron detectors thermocouples, power range neutron flux detector, source range neutron flux detector, and pressure sensors in the plenum between the primary vessel and the SCS. Data from the sensors, scattered around the core vessel of the reactor, is fed into the centralized database, such as DeepLynx, developed by Idaho National Laboratory (INL); processed to the same data format; aggregated by the systems type (nuclear fuel system, primary cooling system, secondary cooling system, power generating system, etc.); and consolidated into the DT of the reactor as a whole. The position of all IoT sensors remains constant and known throughout the reactor's life, and thus, they form a point cloud too, albeit a sparse one. The sparse point cloud of the IoT data cannot be used for a direct prediction of the reactor's state but rather as a verification instrument of the DT model. The data flow for this approach is depicted in Figure 3.

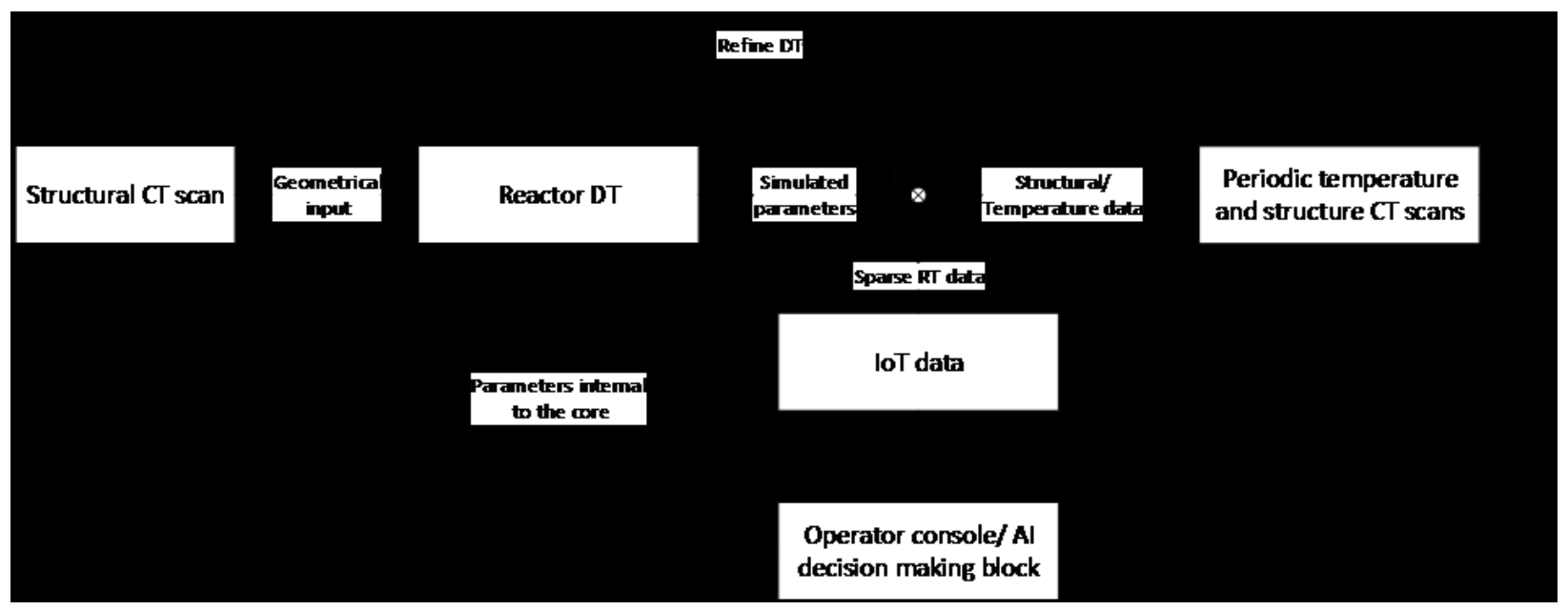

Figure 3. Data flow chart.

The DT of the reactor will be constructed using the simulation code developed at INL: MAMOTH for reactor multiphysics, including structural deformation, and GRIZZLY for reactor materials degradation. The DT will encompass the reactor as a whole, including the fuel, cooling medium, cover gas, and SCS. The DT will be periodically refined using both the data from the regular CT scans, as well as using the real-time data from the IoT sensors. The model discrepancy data will be propagated to the operator's console or potentially an artificial intelligence based decision-making system for analysis. The DT will produce a model of the internal reactor parameters based on available core instrumentation data. These parameters will serve as the input data to generate expected readings of the IoT sensors. It may be necessary to employ a classical machine learning divide et impera approach. Some sensor data will be used to provide input for the DT to predict the output of the other group of sensors. Next, we will compare DT prediction and actual sensor readings. This comparison will provide the quality score of the DT model. 
The desired output of the entire analysis, is the prediction of undesired reactor operation regimes, including those that lead to a potential compromising of the SCS' integrity and structural stability assessment resulting from materials fatigue, creep, or poor craftmanship.

\section{CONCLUSION}

In this study, we proposed a novel microreactor instrumentation concept to assess the integrity of the reactor vessel. The concept focuses on the synergistic utilization of the reactor's ICS sensors, external LDS and thermal cameras, and a DT to deliver information to the operator about the general health of the reactor and particularly about structural integrity of the secondary containment system.

\section{REFERENCES}

Arafat, Yasir. 2020. "Microreactor Applications Research, Validation \& EvaLuation (MARVEL) Project.” Presented at GAIN-NEI-EPRI Microreactor Workshop. Virtual. https://gain.inl.gov/GAINEPRINEI_MicroreactorProgramVirtualWorkshopPres/Day-1\%20Presentati ons/Day-1.05-Arafat_MicroreactorĀpplicationsTesting-MARVEL,18Aug2020.pdf.

Asadi, Khashayar, Hariharan Ramshankar, Mojtaba Noghabaei, and Kevin Han. 2019. "Real-Time Image Localization and Registration with BIM Using Perspective Alignment for Indoor Monitoring of Construction." Journal of Computing in Civil Engineering 33(5): 04019031. https://doi.org/10.1061/(ASCE)CP.1943-5487.0000847.

Bevan, M., and Steve, J. 2016. "LCI National Webinar: Preliminary findings from national project performance research." http://www.leanconstruction.org/media/img/safetyweek/FINAL-2016-05-26_LCI_National_WebinarOwner Project Performance Study Overview and CallToAction.pdf (Nov. 6, 2017).

Bougard, A.J., and B.R. Ellis. 2000. "Laser Measurement of Building Vibration and Displacement." Shock and Vibration 7: 287-298. https://doi.org/10.1155/2000/142757.

Goesele, M., N. Snavely, B. Curless, H. Hoppe, and S. M. Seitz. 2007. "Multi-view stereo for community photo collections." 2007 IEEE 11th Int. Conf. on Computer Vision. Washington D.C.: IEEE Computer Society, 1-8. https://doi.org/10.1109/ICCV.2007.4408933.

Engel, J., T. Schöps, and D. Cremers. 2014. "LSD-SLAM: Large-scale direct monocular SLAM.” In Proc., European Conf. on Computer Vision, 834-849. Dordrecht, Netherlands: Springer.

Ham, Y., Han, K., Lin, J., and Golparvar-Fard, M. 2016. "Visual monitoring of civil infrastructure systems via camera-equipped Unmanned Aerial Vehicles (UAVs): A review of related works." Visualizations in Engineering 4(1): 1-8. https://doi.org/10.1186/s40327-015-0029-z.

Han, K. K., and M. Golparvar-Fard. 2017. "Potential of big visual data and building information modeling for construction performance analytics: An exploratory study." Automation in Construction 73: 184-198. https://doi.org/10.1016/j.autcon.2016.11.004.

Han, Kevin, Joseph Degol, and Mani Golparvar-Fard. 2018. "Geometry- and Appearance-Based Reasoning of Construction Progress Monitoring." Journal of Construction Engineering and Management 144(2): 04017110. https://doi.org/10.1061/(ASCE)CO.1943-7862.0001428.

Kadu, Ajinkya, Hassan Mansour, and Petros T. Boufounos. 2020. "High-Contrast Reflection Tomography with Total-Variation Constraints." IEEE Transactions on Computational Imaging 6: 1523-1536. https://doi.org/10.1109/TCI.2020.3038171.

Wu, C. 2017. "VisualSFM: A Visual Structure from Motion System."2011. http://ccwu.me/vsfm/. 


\section{Appendix A}

\section{Feedback Questionnaire}

- Do you currently employ any digital engineering techniques?

- If yes:

- What are they?

- How successful? (maybe some anecdotal evidence of cost and time saving)

- Did the white paper provide you enough insight about what digital engineering can do for you? If not, what could be further added?

- Would you be willing to explore potential applications of digital engineering to facilitate your small modular reactor design?

- Do you see the value in constructing digital twins of the reactors?

- If yes:

- What critical parameters do they represent?

- What are other important aspects that are omitted in your current DT implementation?

- How does the current implantation of reactor ICS address the need for continuous monitoring of the reactor's critical parameters?

- What is missing in the ICS in terms of sensor equipment?

- How is the sensor data being processed currently?

- Are there any artificial intelligence or machine learning techniques currently employed for sensor data analysis?

- If not - would you be interested in exploring the benefits these techniques can provide?

- Are there any artificial intelligence or machine learning techniques currently employed for reactor control and decision making?

- Is it an option from the regulatory standpoint?

- Would you be willing to collaborate with INL on developing methods to construct DT's of the reactor?

N.B.: Governmental entities (such as National Laboratories) are not allowed to enter into any sort of NDA. Therefore, your response to this questionnaire may be published alongside with this report. Please use your discretion in sharing any potentially sensitive information. 